Research Article

\title{
Monotonicity Analysis of Fractional Proportional Differences
}

\author{
Iyad Suwan, ${ }^{1}$ Shahd Owies, ${ }^{1}$ Muayad Abussa, ${ }^{1}$ and Thabet Abdeljawad $\mathbb{D}^{2,3,4}$ \\ ${ }^{1}$ Department of Mathematics and Statistics, Arab American University, P.O. Box 240 Jenin 13, Zababdeh, State of Palestine \\ ${ }^{2}$ Department of Mathematics and General Sciences, Prince Sultan University, P.O. Box 66833, Riyadh 11586, Saudi Arabia \\ ${ }^{3}$ Department of Medical Research, China Medical University, Taichung 40402, Taiwan \\ ${ }^{4}$ Department of Computer Science and Information Engineering, Asia University, Taichung, Taiwan
}

Correspondence should be addressed to Thabet Abdeljawad; tabdeljawad@psu.edu.sa

Received 18 January 2020; Revised 18 March 2020; Accepted 6 April 2020; Published 1 May 2020

Academic Editor: Manuel De la Sen

Copyright (c) 2020 Iyad Suwan et al. This is an open access article distributed under the Creative Commons Attribution License, which permits unrestricted use, distribution, and reproduction in any medium, provided the original work is properly cited.

\begin{abstract}
In this work, the nabla discrete new Riemann-Liouville and Caputo fractional proportional differences of order $0<\varepsilon<1$ on the time scale $\mathbb{Z}$ are formulated. The differences and summations of discrete fractional proportional are detected on $\mathbb{Z}$, and the fractional proportional sums associated to $\left({ }_{c}^{R} \nabla^{\varepsilon, \rho} \chi\right)(z)$ with order $0<\varepsilon<1$ are defined. The relation between nabla Riemann-Liouville and Caputo fractional proportional differences is derived. The monotonicity results for the nabla Caputo fractional proportional difference are proved; specifically, if $\left({ }_{c-1}^{R} \nabla^{\varepsilon, \rho} \chi\right)(z)>0$ then $\chi(z)$ is $\varepsilon \rho$-increasing, and if $\chi(z)$ is strictly increasing on $\mathbb{N}_{c}$ and $\chi(c)>0$, then $\left({ }_{c-1}^{R} \nabla^{\varepsilon, \rho} \chi\right)(z)>0$. As an application of our findings, a new version of the fractional proportional difference of the mean value theorem (MVT) on $\mathbb{Z}$ is proved.
\end{abstract}

\section{Introduction}

Many problems in science, engineering, and media can be formulated using continuous and discrete fractional calculus [1-14]. The fractional sums and differences and their monotonicity properties are deeply studied in [15-25]. In [26], Atangana and Baleanu solved the fractional heat transfer model using new fractional derivatives with exponential kernels, and they presented many applications of the new notations of fractional derivatives. Applications of discrete fractional calculus are successfully discussed by many researchers in the last decade, for example, in [27-29]. Recently, studying the monotonicity for fractional difference operators with nonsingular discrete kernels is under focus [30, 31]. Monotonicity results for fractional difference operators with discrete exponential kernels were studied in [32] when the time step $h=1$. In [3], deep monotonicity analysis is done for nabla $h$-discrete fractional differences with a discrete Mittag-Leffler kernel in the time scale $h \mathbb{Z}$ with $0<\varepsilon<1$ and $0<h \leq 1$. The results of the research generalized those obtained in [22] where $0<\varepsilon<0.5$ and $h=1$. After that, monotonicity analysis of fractional proportional differences is studied and then the results are prettified by formulating a new version of mean value theorem as an application. In [33], the nabla fractional sums and differences of order $0<\varepsilon<1$ on the time scale $h \mathbb{Z}$ where $0<h \leq 1$ are formulated, and the monotonicity results for the nabla $h$-Caputo fractional difference operator were concluded. In this paper, the authors formulated the nabla discrete new Riemann-Liouville (RL) and Caputo fractional proportional differences of order $0<\varepsilon<1$ on the time scale $\mathbb{Z}$. They also proved a new version of the fractional proportional difference of the mean value theorem (MVT) on $\mathbb{Z}$.

The article is organized as follows: Section 2 presents the main definitions and needed preliminaries. In Section 3, the monotonicity results for fractional proportional differences are classified. In Section 4, we formulate a new version of the mean value theorem as an application. Finally, we provide the conclusions in Section 5.

\section{Definitions and Preliminary Results}

Definition 1. The discrete proportional difference of order $0<\rho \leq 1$ for the function $\chi$ is defined by

$$
\begin{aligned}
\nabla^{\rho} \chi(z) & =(1-\rho) \chi(z)+\rho \nabla \chi(z), \\
z \in \mathbb{N}_{c+1} & =\{c+1, c+2, c+3, \ldots\},
\end{aligned}
$$

and $c \geq 0$ is an integer 
Definition 2. Let $z \in \mathbb{N}_{c}, 0<\rho \leq 1$, and $p=(\rho-1 / \rho)$, then $\widehat{e}_{p}(z, c)=\rho^{z-c}$.

Definition 3. For any real number $\alpha$, the $\alpha$ rising function is $z^{\bar{\alpha}}=(\Gamma(z+\alpha) / \Gamma(z))$, such that $z \in \mathbb{C} \backslash\{\ldots,-2,-1,0\}$, $0^{\bar{\alpha}}=0$, where $\Gamma(z)$ is the gamma function.

Definition 4 (nabla fractional proportional sums).

For a function $\chi: \mathbb{N}_{c} \longrightarrow \mathbb{R}, \rho>0$, and $\varepsilon \in \mathbb{C}$, $0<\operatorname{Re}(\varepsilon)<1$, the nabla left fractional proportional sum of $\chi$ starting at $c$ is defined by

$$
\begin{aligned}
\left({ }_{c} \nabla^{-\varepsilon, \rho} \chi\right)(z) & =\frac{1}{\Gamma(\varepsilon)} \int_{c}^{z} \widehat{e}_{p}(z-s, 0)(z-\varsigma(s))^{\overline{\varepsilon-1}} \chi(s) \nabla s \\
& =\frac{1}{\Gamma(\varepsilon)} \sum_{l=c+1}^{z} \widehat{e}_{p}(z-\iota, 0)(z-\varsigma(\iota))^{\overline{\varepsilon-1}} \chi(\iota), \quad z \in \mathbb{N}_{c} .
\end{aligned}
$$

For the function $\chi:{ }_{d} \mathbb{N}=\{d, d-1, d-2, \ldots\} \rightarrow \mathbb{R}$, the nabla right fractional proportional sum ending at $d$ is defined by

$$
\begin{aligned}
\left(\nabla_{d}^{-\varepsilon, \rho} \chi\right)(z) & =\frac{1}{\Gamma(\varepsilon)} \int_{z}^{d} \widehat{e}_{p}(s-z, 0)(s-\varsigma(z))^{\overline{\varepsilon-1}} \chi(s) \Delta s \\
& =\frac{1}{\Gamma(\varepsilon)} \sum_{\imath=z}^{d-1} \widehat{e}_{p}(\iota-z, 0)(\iota-\varsigma(z))^{\overline{\varepsilon-1}} \chi(\iota), \quad z \epsilon_{d} \mathbb{N} .
\end{aligned}
$$

We notice that by setting $\rho=1$, the given definitions of the fractional sums are generalizations of the Riemann fractional sums.

Lemma 1. Let $\chi: \mathbb{N}_{c} \longrightarrow \mathbb{R}$ be a function, $p=(\rho-1 / \rho)$, $0<\varepsilon<1$, and $0<\rho \leq 1$, then

$$
\begin{aligned}
& \left({ }_{c} \nabla^{-\varepsilon, \rho} \nabla \chi\right)(z) \\
& \quad=\left(\nabla_{c} \nabla^{-\varepsilon, \rho} \chi\right)(z)-\frac{(z-c)^{\overline{\varepsilon-1}}}{\Gamma(\varepsilon)} \widehat{e}_{p}(z-1, c) \chi(c) .
\end{aligned}
$$

Proof.

$$
\begin{aligned}
& \left({ }_{c} \nabla^{-\varepsilon, \rho} \nabla \chi\right)(z)=\frac{1}{\Gamma(\varepsilon)} \sum_{\iota=c+1}^{z} \widehat{e}_{p}(z-\iota, 0)(z-\varsigma(\iota))^{\overline{\varepsilon-1}} \nabla \chi(\iota) \\
& =\frac{1}{\Gamma(\varepsilon)} \sum_{\iota=c+1}^{z} \rho^{z-\iota}(z-\varsigma(\iota))^{\overline{\varepsilon-1}}(\chi(\iota)-\chi(\iota-1)) \\
& =\frac{1}{\Gamma(\varepsilon)} \sum_{\iota=c+1}^{z} \rho^{z-\iota}(z-\varsigma(\iota))^{\overline{\varepsilon-1}} \chi(\iota) \\
& -\frac{1}{\Gamma(\varepsilon)} \sum_{\iota=c+1}^{z} \rho^{z-\iota}(z-\varsigma(\iota))^{\overline{\varepsilon-1}} \chi(\iota-1) \\
& =\frac{1}{\Gamma(\varepsilon)} \sum_{\iota=c+1}^{z} \rho^{z-\iota}(z-\varsigma(\iota))^{\overline{\varepsilon-1}} \chi(\iota) \\
& -\frac{1}{\Gamma(\varepsilon)} \sum_{\imath=c}^{z-1} \rho^{z-\iota-1}(z-\varsigma(\iota+1))^{\overline{\varepsilon-1}} \chi(\iota) \\
& =\frac{1}{\Gamma(\varepsilon)}\left(\sum_{\iota=c+1}^{z} \rho^{z-\iota}(z-\varsigma(\iota))^{\overline{\varepsilon-1}} \chi(\iota)-\sum_{\iota=c}^{z-1} \rho^{(z-1)-\iota}((z-1)-\varsigma(\iota))^{\bar{\varepsilon}-1} \chi(\iota)\right) \\
& =\frac{1}{\Gamma(\varepsilon)}\left(\sum_{\iota=c+1}^{z} \rho^{z-\iota}(z-\varsigma(\iota))^{\overline{\varepsilon-1}} \chi(\iota)-\sum_{\iota=c+1}^{z-1} \rho^{(z-1)-\iota}((z-1)-\varsigma(\iota))^{\overline{\varepsilon-1}} \chi(\iota)\right) \\
& =\frac{1}{\Gamma(\varepsilon)} \rho^{(z-1)-c}((z-1)-\varsigma(c))^{\overline{\varepsilon-1}} \chi(c) \\
& =\frac{1}{\Gamma(\varepsilon)} \nabla \sum_{\iota=c+1}^{z} \rho^{z-\iota}(z-\varsigma(\iota))^{\overline{\varepsilon-1}} \chi(\iota)-\frac{1}{\Gamma(\varepsilon)} \rho^{(z-1)-c}(z-1-c+1)^{\overline{\varepsilon-1}} \chi(c) \\
& =\left(\nabla_{c} \nabla^{-\varepsilon, \rho} \chi\right)(z)-\frac{(z-c)^{\overline{\varepsilon-1}}}{\Gamma(\varepsilon)} \widehat{e}_{p}(z-1, c) \chi(c) .
\end{aligned}
$$


Lemma 2. Let $\chi: \mathbb{N}_{c} \longrightarrow \mathbb{R}, p=(\rho-1 / \rho), 0<\varepsilon<1$, and Proof. $0<\rho \leq 1$, then

$$
\left({ }_{c} \nabla^{-\varepsilon, \rho} \nabla^{\rho} \chi\right)(z)=\left(\nabla_{c}^{\rho} \nabla^{-\varepsilon, \rho} \chi\right)(z)-\frac{\rho}{\Gamma(\varepsilon)}(z-c)^{\overline{\varepsilon-1}} \widehat{e}_{p}(z-1, c) \chi(c) .
$$

$$
\nabla^{\rho} \chi(z)=(1-\rho) \chi(z)+\rho \nabla \chi(z)
$$

hence,

$$
\begin{aligned}
\left({ }_{c} \nabla^{-\varepsilon, \rho} \nabla^{\rho} \chi\right)(z) & ={ }_{c} \nabla^{-\varepsilon, \rho}((1-\rho) \chi(z)+\rho \nabla \chi(z)) \\
& =(1-\rho)\left({ }_{c} \nabla^{-\varepsilon, \rho} \chi\right)(z)+\rho\left({ }_{c} \nabla^{-\varepsilon, \rho} \nabla \chi(z)\right) \text { using Lemma } 1 \\
& =(1-\rho)\left({ }_{c} \nabla^{-\varepsilon, \rho} \chi\right)(z)+\rho\left(\left(\nabla^{\rho}{ }_{c} \nabla^{-\varepsilon, \rho} \chi\right)(z)-\frac{(z-c)^{\overline{\varepsilon-1}}}{\Gamma(\varepsilon)} \widehat{e}_{p}(z-1, c) \chi(c)\right) \\
& =\left((1-\rho)\left({ }_{c} \nabla^{-\varepsilon, \rho} \chi\right)(z)+\rho \nabla^{\rho}\left({ }_{c} \nabla^{-\varepsilon, \rho} \chi\right)(z)\right)-\frac{\rho}{\Gamma(\varepsilon)}(z-c)^{\overline{\varepsilon-1}} \widehat{e}_{p}(z-1, c) \chi(c) \\
& =\left(\nabla_{c}^{\rho} \nabla^{-\varepsilon, \rho} \chi\right)(z)-\frac{\rho}{\Gamma(\varepsilon)}(z-c)^{\frac{\varepsilon-1}{{ }^{\varepsilon-1}}} \widehat{e}_{p}(z-1, c) \chi(c) .
\end{aligned}
$$

Note that if $\rho=1$, we get

$$
\left({ }_{c} \nabla^{-\varepsilon} \nabla \chi\right)(z)=\left(\nabla_{c} \nabla^{-\varepsilon} \chi\right)(z)-\frac{1}{\Gamma(\varepsilon)}(z-c)^{\overline{\varepsilon-1}} \chi(c) \text {. }
$$

Definition 5 (Riemann-Liouville (RL) fractional proportional differences)

For $0<\rho \leq 1, \varepsilon \in \mathbb{C}, 0<\operatorname{Re}(\varepsilon)<1$, and $\chi$ be a function defined on $\mathbb{N}_{c}$ or on ${ }_{d} \mathbb{N}$, then the left Riemann-Liouville fractional proportional difference starting at $c$ is defined by

$$
\begin{aligned}
\left({ }_{c}^{R} \nabla^{\varepsilon, \rho} \chi\right)(z) & =\nabla_{c}^{\rho} \nabla^{-(1-\varepsilon), \rho} \chi(z) \\
& =\frac{\nabla^{\rho}}{\Gamma(1-\varepsilon)} \int_{c}^{z} \widehat{e}_{p}(z-s, 0)(z-\varsigma(s))^{\overline{-\varepsilon}} \chi(s) \nabla s \\
& =\frac{\nabla^{\rho}}{\Gamma(1-\varepsilon)} \sum_{\iota=c+1}^{z} \widehat{e}_{p}(z-\iota, 0)(z-\varsigma(\iota))^{\overline{-\varepsilon}} \chi(\iota) \\
& =\frac{\nabla^{\rho}}{\Gamma(1-\varepsilon)} \sum_{\iota=c+1}^{z} \rho^{z-\iota}(z-\varsigma(\iota))^{\overline{-\varepsilon}} \chi(\iota)
\end{aligned}
$$

and the right Riemann-Liouville fractional proportional difference ending at $d$ is defined by

$$
\begin{aligned}
\left({ }^{R} \nabla_{d}^{\varepsilon, \rho} \chi\right)(z) & =-\Delta^{\rho} \nabla_{d}^{-(1-\varepsilon), \rho} \chi(z) \\
& =\frac{-\Delta^{\rho}}{\Gamma(1-\varepsilon)} \sum_{l=z}^{d-1} \rho^{\imath-z}(\iota-\varsigma(z))^{-\bar{\varepsilon}} \chi(\iota) .
\end{aligned}
$$

We notice that by setting $\rho=1$, the given definitions of the fractional differences are generalizations of the Riemann fractional differences.
Definition 6 (Caputo fractional proportional differences)

For $0<\rho \leq 1, \varepsilon \in \mathbb{C}, 0<\operatorname{Re}(\varepsilon)<1$, and $\chi$ be a function defined on $\mathbb{N}_{c}$ or on ${ }_{d} \mathbb{N}$, then the left Caputo fractional proportional difference starting at $c$ is defined by

$$
\begin{aligned}
\left({ }_{c}^{C} \nabla^{\varepsilon, \rho} \chi\right)(z) & ={ }_{c} \nabla^{-(1-\varepsilon), \rho} \nabla^{\rho} \chi(z) \\
& =\frac{1}{\Gamma(1-\varepsilon)} \int_{c}^{z} \widehat{e}_{p}(z-s, 0)(z-\varsigma(s))^{\overline{-\varepsilon}}\left(\nabla^{\rho} \chi(s)\right) \nabla s \\
& =\frac{1}{\Gamma(1-\varepsilon)} \sum_{\iota=c+1}^{z} \rho^{z-\iota}(z-\varsigma(\iota))^{-\bar{\varepsilon}} \nabla^{\rho} \chi(\iota),
\end{aligned}
$$

and the right Caputo fractional proportional difference ending at $d$ is defined by

$$
\begin{aligned}
\left({ }^{C} \nabla_{d}^{\varepsilon, \rho} \chi\right)(z) & =\nabla_{d}^{-(1-\varepsilon), \rho}\left(-\Delta^{\rho} \chi(z)\right) \\
& =\frac{1}{\Gamma(1-\varepsilon)} \sum_{l=z}^{d-1} \rho^{\iota-z}(\iota-\varsigma(z))^{\overline{-\varepsilon}}\left(-\Delta^{\rho} \chi(\iota)\right) .
\end{aligned}
$$

We notice that by setting $\rho=1$, the given definitions of the fractional differences are generalizations of the Caputo fractional differences.

Proposition 1 (the relation between nabla RL and Caputo fractional proportional differences)

For any $\varepsilon \in \mathbb{C}, 0<\operatorname{Re}(\varepsilon)<1$, and $0<\rho \leq 1$, the relation between nabla $R L$ and Caputo fractional proportional differences is given as follows: 
(i) $\left({ }_{c}^{C} \nabla^{\varepsilon, \rho} \chi\right)(z)=\left({ }_{c}^{R} \nabla^{\varepsilon, \rho} \chi\right)(z)-(z-c)^{\overline{-\varepsilon}} / \Gamma(1-\varepsilon) \widehat{e}_{p}(z, \quad$ Proof. c) $\chi(c)$.

(ii) $\left({ }_{c}^{C} \nabla^{\varepsilon, \rho} \chi\right)(z)=\left({ }_{c}^{R} \nabla^{\varepsilon, \rho} \chi\right)(z)-(d-z)^{\overline{-\varepsilon}} / \Gamma(1-\varepsilon) \widehat{e}_{p}(d$, $z) \chi(d)$.

$$
\begin{aligned}
& \left({ }_{c}^{C} \nabla^{\varepsilon, \rho} \chi\right)(z)=\frac{1}{\Gamma(1-\varepsilon)} \sum_{\iota=c+1}^{z} \rho^{z-\iota}(z-\varsigma(\iota))^{-\bar{\varepsilon}} \nabla^{\rho} \chi(\iota) \\
& =\frac{1}{\Gamma(1-\varepsilon)} \sum_{l=c+1}^{z} \rho^{z-\iota}(z-\varsigma(\iota))^{\overline{-\varepsilon}}((1-\rho) \chi(\iota)+\rho \nabla \chi(\iota)) \\
& =\frac{1}{\Gamma(1-\varepsilon)} \sum_{\iota=c+1}^{z} \rho^{z-\iota}(z-\varsigma(\iota))^{-\bar{\varepsilon}}(1-\rho) \chi(\iota) \\
& +\frac{1}{\Gamma(1-\varepsilon)} \sum_{\iota=c+1}^{z} \rho^{z-\iota}(z-\varsigma(\iota))^{\overline{-\varepsilon}}(\rho \nabla \chi(\iota)) \\
& =\frac{1}{\Gamma(1-\varepsilon)} \sum_{\iota=c+1}^{z} \rho^{z-\iota}(z-\varsigma(\iota))^{\overline{-\varepsilon}}(1-\rho) \chi(\iota) \\
& +\frac{1}{\Gamma(1-\varepsilon)} \sum_{\iota=c+1}^{z} \rho^{z-\iota}(z-\varsigma(\iota))^{\overline{-\varepsilon}} \rho(\chi(\iota)-\chi(\iota-1)) \\
& =\frac{1}{\Gamma(1-\varepsilon)} \sum_{\iota=c+1}^{z} \rho^{z-\iota}(z-\varsigma(\iota))^{\overline{-\varepsilon}}(1-\rho) \chi(\iota) \\
& +\frac{1}{\Gamma(1-\varepsilon)} \sum_{\iota=c+1}^{z} \rho^{z-\iota}(z-\varsigma(\iota))^{-\bar{\varepsilon}} \rho \chi(\iota) \\
& -\frac{1}{\Gamma(1-\varepsilon)} \sum_{l=c}^{z-1} \rho^{z-\imath-1}(z-\varsigma(\iota+1))^{\overline{-\varepsilon}} \rho \chi(k) \\
& =\frac{1-\rho}{\Gamma(1-\varepsilon)} \sum_{\iota=c+1}^{z} \rho^{z-\iota}(z-\varsigma(\iota))^{\overline{-\varepsilon}} \chi(\iota) \\
& +\frac{\rho}{\Gamma(1-\varepsilon)} \sum_{\iota=c+1}^{z} \rho^{z-\iota}(z-\varsigma(\iota))^{\overline{-\varepsilon}} \chi(\iota) \\
& -\frac{\rho}{\Gamma(1-\varepsilon)} \sum_{\iota=c+1}^{z-1} \rho^{z-1-\iota}(z-1-\varsigma(\iota))^{-\bar{\varepsilon}} \chi(\iota) \\
& -\frac{\rho}{\Gamma(1-\varepsilon)} \rho^{z-1-c}(z-1-\varsigma(c))^{\overline{-\varepsilon}} \chi(c) \\
& =\frac{1-\rho}{\Gamma(1-\varepsilon)} \sum_{\iota=c+1}^{z} \rho^{z-\iota}(z-\varsigma(\iota))^{\overline{-\varepsilon}} \chi(\iota)+\frac{\rho \nabla}{\Gamma(1-\varepsilon)} \sum_{\iota=c+1}^{z} \rho^{z-\iota}(z-\varsigma(\iota))^{\overline{-\varepsilon}} \chi(\iota) \\
& -\frac{\rho}{\Gamma(1-\varepsilon)} \rho^{z-1-c}(z-1-c+1)^{-\bar{\varepsilon}} \chi(c)
\end{aligned}
$$




$$
\begin{aligned}
= & \frac{\nabla^{\rho}}{\Gamma(1-\varepsilon)} \sum_{l=c+1}^{z} \widehat{e}_{p}(z-\iota, 0)(z-\varsigma(\iota))^{-\bar{\varepsilon}} \chi(\iota) \\
& -\frac{(z-c)^{-\varepsilon}}{\Gamma(1-\varepsilon)} \rho^{z-c} \chi(c)=\left({ }_{c}^{C} \nabla^{\varepsilon, \rho} \chi\right)(z)-\frac{(z-c)^{-\bar{\varepsilon}}}{\Gamma(1-\varepsilon)} \widehat{e}_{p}(z, c) \chi(c) .
\end{aligned}
$$

$$
\begin{aligned}
& \left({ }^{C} \nabla_{d}^{\varepsilon, \rho} \chi\right)(z)=\frac{1}{\Gamma(1-\varepsilon)} \sum_{l=z}^{d-1} \rho^{\iota-z}(\iota-\varsigma(z))^{-\bar{\varepsilon}}\left(-\Delta^{\rho}\right) \chi(\iota) \\
& =\frac{-1}{\Gamma(1-\varepsilon)} \sum_{l=z}^{d-1} \rho^{\iota-z}(\iota-\varsigma(z))^{\overline{-\varepsilon}}((1-\rho) \chi(\iota)+\rho \Delta \chi(\iota)) \\
& =\frac{-1}{\Gamma(1-\varepsilon)} \sum_{\imath=z}^{d-1} \rho^{\iota-z}(\iota-\varsigma(z))^{-\bar{\varepsilon}}(1-\rho) \chi(\iota) \\
& -\frac{1}{\Gamma(1-\varepsilon)} \sum_{\iota=z}^{d-1} \rho^{\iota-z}(\iota-\varsigma(z))^{\bar{\varepsilon}} \rho(\chi(\iota+1)-\chi(\iota)) \\
& =\frac{-1}{\Gamma(1-\varepsilon)} \sum_{\imath=z}^{d-1} \rho^{\imath-z}(\iota-\varsigma(z))^{\overline{-\varepsilon}}(1-\rho) \chi(\iota) \\
& -\frac{1}{\Gamma(1-\varepsilon)} \sum_{l=z}^{d-1} \rho^{\iota-z}(\iota-\varsigma(z))^{\bar{\varepsilon}} \rho \chi(\iota+1)+\frac{1}{\Gamma(1-\varepsilon)} \sum_{\iota=z}^{d-1} \rho^{\iota-z}(\iota-\varsigma(z))^{\overline{-\varepsilon}} \rho \chi(\iota) \\
& =\frac{-1}{\Gamma(1-\varepsilon)} \sum_{l=z}^{d-1} \rho^{l-z}(\iota-\varsigma(z))^{-\bar{\varepsilon}}(1-\rho) \chi(\iota) \\
& -\frac{1}{\Gamma(1-\varepsilon)} \sum_{\iota=z+1}^{d} \rho^{\iota-1-z}(\iota-1-\varsigma(z))^{\overline{-\varepsilon}} \rho \chi(\iota) \\
& +\frac{1}{\Gamma(1-\varepsilon)} \sum_{\iota=z}^{d-1} \rho^{\iota-z}(\iota-\varsigma(z))^{\overline{-\varepsilon}} \rho \chi(\iota) \\
& =\frac{-1}{\Gamma(1-\varepsilon)} \sum_{\imath=z}^{d-1} \rho^{\iota-z}(\iota-\varsigma(z))^{-\bar{\varepsilon}}(1-\rho) \chi(\iota) \\
& -\frac{1}{\Gamma(1-\varepsilon)} \rho^{d-1-z}(d-1-\varsigma(z))^{\overline{-\varepsilon}} \rho \chi(d) \\
& -\frac{1}{\Gamma(1-\varepsilon)} \sum_{l=z+1}^{d-1} \rho^{l-(z+1)}(\iota-\varsigma(z+1))^{-\bar{\varepsilon}} \rho \chi(\iota) \\
& +\frac{1}{\Gamma(1-\varepsilon)} \sum_{l=z}^{d-1} \rho^{\imath-z}(\iota-\varsigma(z))^{\overline{-\varepsilon}} \rho \chi(\iota) \\
& =\frac{-(1-\rho)}{\Gamma(1-\varepsilon)} \sum_{\imath=z}^{d-1} \rho^{\iota-z}(\iota-\varsigma(z))^{\overline{-\varepsilon}} \chi(\iota)-\frac{\rho \Delta}{\Gamma(1-\varepsilon)} \sum_{l=z}^{d-1} \rho^{\iota-z}(\iota-\varsigma(z))^{\overline{-\varepsilon}} \rho \chi(\iota) \\
& -\frac{1}{\Gamma(1-\varepsilon)} \rho^{d-z}(d-1-z+1)^{-\bar{\varepsilon}} \chi(d) \\
& =\frac{-\Delta^{\rho}}{\Gamma(1-\varepsilon)} \sum_{l=z}^{d-1} \rho^{l-z}(\iota-\varsigma(z))^{\overline{-\varepsilon}} \chi(\iota)-\frac{1}{\Gamma(1-\varepsilon)} \rho^{d-z}(d-z)^{\overline{-\varepsilon}} \chi(d) \\
& =\left({ }^{R} \nabla_{d}^{\varepsilon, \rho} \chi\right)(z)-\frac{(d-z)^{\overline{-\varepsilon}}}{\Gamma(1-\varepsilon)} \widehat{e}_{p}(d, z) \chi(d) .
\end{aligned}
$$


Numerical calculations have been done in order to verify the first equation in Proposition 1. The values used are $c=2.5, \rho=0.7$, and $\varepsilon=0.3$. The results are illustrated in Figure 1 .

In addition to that, the data are presented in Table 1.

Lemma 3. Let $0<\varepsilon<1, \quad z, \iota \in \mathbb{N}_{c}$, and $\iota<z$, then $\nabla(z-\varsigma(\iota))^{\overline{-\varepsilon}}=-\varepsilon(z-\varsigma(\iota))^{\overline{-\varepsilon-1}}$.

Proof.

$$
\begin{aligned}
\nabla(z-\varsigma(\iota))^{\overline{-\varepsilon}} & =(z-\varsigma(\iota))^{\overline{-\varepsilon}}-(z-1-\varsigma(\iota))^{\overline{-\varepsilon}} \\
& =(z-\iota+1)^{\overline{-\varepsilon}}-(z-\iota)^{\overline{-\varepsilon}} \\
& =\frac{\Gamma(z-\iota-\varepsilon+1)}{\Gamma(z-\iota+1)}-\frac{\Gamma(z-\iota-\varepsilon)}{\Gamma(z-\iota)} \\
& =\frac{\Gamma(z-\iota-\varepsilon)}{\Gamma(z-\iota)}\left(\frac{z-\iota-\varepsilon}{z-\iota}-1\right) \\
& =\frac{\Gamma(z-\iota+1-\varepsilon-1)}{\Gamma(z-\iota)}\left(\frac{-\varepsilon}{z-\imath}\right) \\
& =-\varepsilon \frac{\Gamma(z-\iota+1-\varepsilon-1)}{\Gamma(z-\iota+1)} \\
& =-\varepsilon(z-\iota+1)^{\overline{-\varepsilon-1}}=-\varepsilon(z-\varsigma(\iota))^{\overline{-\varepsilon-1}} .
\end{aligned}
$$

\section{Monotonicity Results}

The following two monotonicity definitions are given in [18].

Definition 7. Let $y: \mathbb{N}_{a} \longrightarrow \mathbb{R}$ be a function satisfying $y(a) \geq 0,0<\alpha<1$. Then, $y(t)$ is called an $\alpha$-increasing function on $\mathbb{N}_{a}$ if $y(t+1) \geq \alpha y(t) \quad \forall t \in \mathbb{N}_{a}$.

Definition 8. Let $y: \mathbb{N}_{a} \longrightarrow \mathbb{R}$ be a function satisfying $y(a) \leq 0,0<\alpha<1$. Then, $y(t)$ is called an $\alpha$-decreasing function on $\mathbb{N}_{a}$ if $y(t+1) \leq \alpha y(t) \forall t \in \mathbb{N}_{a}$.

In the following, we report the new proportional monotonicity main results.

Theorem 1. Let $\chi: \mathbb{N}_{c} \longrightarrow \mathbb{R}$ be a function, and suppose that $\left({ }_{c-1}^{R} \nabla^{\varepsilon, \rho} \chi\right)(z) \geq 0$ for $0<\varepsilon<1$ and $0<\rho \leq 1, z \in \mathbb{N}_{c-1}$. Then, $\chi(z)$ is $\varepsilon \rho-$ increasing.

Proof.

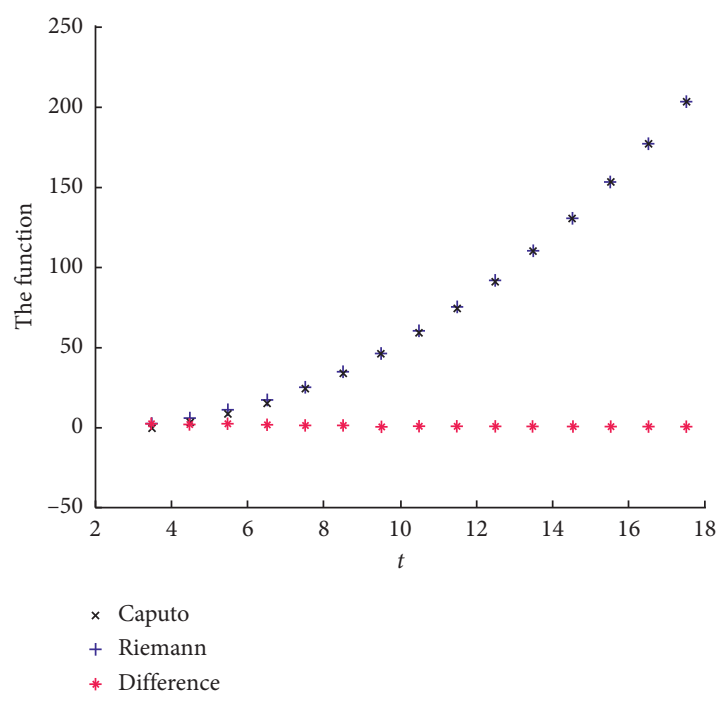

Figure 1: The relation between nabla Riemann and Caputo fractional proportional differences.

TABLE 1: The relation between nabla Riemann and Caputo fractional proportional differences.

\begin{tabular}{lccc}
\hline$z$ & Caputo & Riemann & Difference \\
\hline 3.5 & 2.181077 & -0.415443 & 2.596520 \\
4.5 & 5.786530 & 3.190010 & 2.596520 \\
5.5 & 10.787558 & 8.649248 & 2.138310 \\
6.5 & 17.274639 & 15.611509 & 1.663130 \\
7.5 & 25.339691 & 24.081105 & 1.258585 \\
8.5 & 35.058768 & 34.121524 & 0.937244 \\
9.5 & 46.490945 & 45.800344 & 0.690601 \\
10.5 & 59.680789 & 59.175722 & 0.505066 \\
11.5 & 74.661373 & 74.294052 & 0.367321 \\
12.5 & 91.456990 & 91.190999 & 0.265991 \\
13.5 & 110.085354 & 109.893402 & 0.191952 \\
14.5 & 130.559312 & 130.421178 & 0.138134 \\
15.5 & 152.888147 & 152.788974 & 0.099173 \\
16.5 & 177.078543 & 177.007482 & 0.071061 \\
17.5 & 203.135301 & 203.084469 & 0.050832 \\
\hline
\end{tabular}

$$
\begin{aligned}
\left({ }_{c-1}^{R} \nabla^{\varepsilon, \rho} \chi\right)(z) & =\frac{\nabla^{\rho}}{\Gamma(1-\varepsilon)} \sum_{l=c}^{z} \widehat{e}_{p}(z-\iota, 0)(z-\varsigma(\iota))^{\overline{-\varepsilon}} \chi(\iota) \\
& =\frac{\nabla^{\rho}}{\Gamma(1-\varepsilon)} \sum_{l=c}^{z} \rho^{z-\iota}(z-\varsigma(\iota))^{\overline{-\varepsilon}} \chi(\iota) .
\end{aligned}
$$

Let

$$
S(z)=\sum_{\iota=c}^{z} \rho^{z-\iota}(z-\varsigma(\iota))^{\overline{-\varepsilon}} \chi(\iota) .
$$

Then,

$$
\left({ }_{c-1}^{R} \nabla^{\varepsilon, \rho} \chi\right)(z)=\frac{\nabla^{\rho}}{\Gamma(1-\varepsilon)} S(z)
$$

Hence, from the assumption, we have $\nabla^{\rho} S(z) \geq 0$. That is, 


$$
\begin{array}{rlrl}
\nabla^{\rho} S(z)= & (1-\rho) S(z)+\rho \nabla S(z) & \chi(c+1) & \geq \frac{\varepsilon}{\Gamma(1-\varepsilon)} \sum_{\iota=c}^{c} \rho^{c+1-\iota} \chi(\iota)(c+1-\varsigma(\iota))^{\overline{-\varepsilon-1}} \\
= & (1-\rho) S(z)+\rho(S(z)-S(z-1)) & =\frac{\varepsilon}{\Gamma(1-\varepsilon)} \rho^{c+1-c} \chi(c)(c+1-\varsigma(c))^{\overline{-\varepsilon-1}} \\
= & S(z)-\rho S(z)+\rho S(z)-\rho S(z-1) & & \frac{\varepsilon}{\Gamma(1-\varepsilon)} \rho \chi(c)(c+1-c+1)^{\overline{-\varepsilon-1}} \\
= & S(z)-\rho S(z-1) & =\frac{\varepsilon \rho}{\Gamma(1-\varepsilon)} \chi(c) \frac{\Gamma(1-\varepsilon)}{\Gamma(2)} \\
= & \sum_{\iota=c}^{z} \rho^{z-\iota}(z-\varsigma(\iota))^{\bar{\varepsilon}} \chi(\iota) & =\varepsilon \rho \chi(c) .
\end{array}
$$$$
=(z-\varsigma(z))^{\overline{-\varepsilon}} \chi(z)+\sum_{\imath=c}^{z-1} \rho^{z-\iota}(z-\varsigma(\iota))^{\overline{-\varepsilon}} \chi(\iota)
$$$$
-\sum_{\imath=c}^{z-1} \rho^{z-1-\iota}(z-1-\varsigma(\iota))^{-\varepsilon} \chi(\iota)
$$$$
=(z-z+1)^{\overline{-\varepsilon}} \chi(z)+\sum_{\imath=c}^{z-1} \rho^{z-\iota} \chi(\iota)
$$$$
\cdot\left((z-\varsigma(\iota))^{\bar{\varepsilon}}-(z-1-\varsigma(\iota))^{\bar{\varepsilon}}\right)
$$$$
=(1)^{\overline{-\varepsilon}} \chi(z)+\sum_{l=c}^{z-1} \rho^{z-\iota} \chi(\iota) \nabla(z-\varsigma(\iota))^{\overline{-\varepsilon}}
$$$$
=\frac{\Gamma(1-\varepsilon)}{\Gamma(1)} \chi(z)+\sum_{\iota=c}^{z-1} \rho^{z-\iota} \chi(\iota)\left(-\varepsilon(z-\varsigma(\iota))^{\overline{-\varepsilon-1}}\right)
$$$$
=\Gamma(1-\varepsilon) \chi(z)-\varepsilon \sum_{\imath=c}^{z-1} \rho^{z-\iota} \chi(\iota)(z-\varsigma(\iota))^{\overline{-\varepsilon-1}}
$$$$
\geq 0 \text {. }
$$

Therefore,

$$
\begin{aligned}
\left(c_{c-1}^{R} \nabla^{\varepsilon, \rho} \chi\right)(z) & =\frac{\nabla^{\rho}}{\Gamma(1-\varepsilon)} S(z) \\
& =\frac{1}{\Gamma(1-\varepsilon)}\left(\Gamma(1-\varepsilon) \chi(z)-\varepsilon \sum_{l=c}^{z-1} \rho^{z-\imath} \chi(\iota)(z-\varsigma(\iota))^{-\varepsilon-1}\right) \\
& =\chi(z)-\frac{\varepsilon}{\Gamma(1-\varepsilon)} \sum_{l=c}^{z-1} \rho^{z-t} \chi(\iota)(z-\varsigma(\iota))^{-\varepsilon-1} \\
& =0 .
\end{aligned}
$$

Hence,

$$
\chi(z) \geq \frac{\varepsilon}{\Gamma(1-\varepsilon)} \sum_{l=c}^{z-1} \rho^{z-\iota} \chi(\iota)(z-\varsigma(\iota))^{\overline{-\varepsilon-1}} .
$$

Clearly, $\chi(c-1)=0$. So, we can start the induction from the next step. When $z=c$, we get $\chi(c) \geq 0$; also, when $z=c+1$, we have

Now for $z+1$, replace $z$ by $z+1$, then we get

$$
\begin{aligned}
\chi(z+1) & \geq \frac{\varepsilon}{\Gamma(1-\varepsilon)} \sum_{\iota=c}^{z} \rho^{z+1-\iota} \chi(\iota)(z+1-\varsigma(\iota))^{\overline{\varepsilon-1}} \\
& \geq \frac{\varepsilon}{\Gamma(1-\varepsilon)} \rho^{z+1-z} \chi(z)(z+1-\varsigma(z))^{\overline{-\varepsilon-1}} \\
& =\frac{\varepsilon}{\Gamma(1-\varepsilon)} \rho \chi(z)(z+1-z+1)^{\overline{-\varepsilon-1}} \\
& =\frac{\varepsilon \rho}{\Gamma(1-\varepsilon)} \chi(z) 2^{-\varepsilon-1} \\
& =\varepsilon \rho \chi(z) .
\end{aligned}
$$

Hence, $\chi(z)$ is $\varepsilon \rho$-increasing which completes the proof.

Using Theorem 1 and Proposition 1 we can state the following Caputo fractional proportional difference monotonicity result.

Corollary 1. Let $\chi: \mathbb{N}_{c-1} \longrightarrow \mathbb{R}$ be a function, and suppose that for $0<\varepsilon<1$ and $0<\rho \leq 1$. Suppose that

$$
\left({ }_{c-1}^{C} \nabla^{\varepsilon, \rho} \chi\right)(z) \geq \frac{-\widehat{e}_{p}(z, c-1)}{\Gamma(1-\varepsilon)}(z-c+1)^{\overline{-}} \chi(c-1), \quad z \in \mathbb{N}_{c-1},
$$

then $\chi(z)$ is $\varepsilon \rho-$ increasing.

Proof.

$$
\begin{aligned}
\left({ }_{c-1}^{C} \nabla^{\varepsilon, \rho} \chi\right)(z)= & \left({ }_{c-1}^{R} \nabla^{\varepsilon, \rho} \chi\right)(z)-\frac{\widehat{e}_{p}(z, c-1)}{\Gamma(1-\varepsilon)} \\
& \cdot(z-c+1)^{-\bar{\varepsilon}} \chi(c-1), \quad \forall z \in \mathbb{N}_{c-1},
\end{aligned}
$$

now, from the assumption we have

$$
\begin{array}{r}
\left({ }_{c-1}^{C} \nabla^{\varepsilon, \rho} \chi\right)(z) \geq \frac{-\widehat{e}_{p}(z, c-1)}{\Gamma(1-\varepsilon)}(z-c+1)^{-\bar{\varepsilon}} \chi(c-1), \\
z \in \mathbb{N}_{c-1}, z \in \mathbb{N}_{c-1},
\end{array}
$$

hence, 


$$
\begin{array}{r}
\left({ }_{c-1}^{C} \nabla^{\varepsilon, \rho} \chi\right)(z)+\frac{\widehat{e}_{p}(z, c-1)}{\Gamma(1-\varepsilon)}(z-c+1)^{\overline{-\varepsilon}} \chi(c-1) \geq 0, \\
z \in \mathbb{N}_{c-1}, z \in \mathbb{N}_{c-1},
\end{array}
$$

which means that $\left({ }_{c-1}^{R} \nabla^{\varepsilon, \rho} \chi\right)(z) \geq 0$.

Now, from Theorem 1, we conclude that $\chi(z)$ is $\varepsilon \rho-$ increasing.

Theorem 2. Let $\chi: \mathbb{N}_{c-1} \longrightarrow \mathbb{R}$ be a function which satisfies $\chi(c) \geq 0$, and suppose that for $0<\varepsilon<1$ and $0<\rho \leq 1$. If $\chi(z)$ is increasing on $\mathbb{N}_{c}$, then we have

$$
\left({ }_{c-1}^{R} \nabla^{\mathcal{\varepsilon}, \rho} \chi\right)(z) \geq 0, \quad \forall z \in \mathbb{N}_{c-1} .
$$

Proof. Since

$$
\begin{array}{r}
\left({ }_{c-1}^{R} \nabla^{\varepsilon, \rho} \chi\right)(z)=\chi(z)-\frac{\varepsilon}{\Gamma(1-\varepsilon)} \sum_{\imath=c}^{z-1} \rho^{z-\iota}(z-\varsigma(\iota))^{\overline{-\varepsilon-1}} \chi(\iota), \\
z \in \mathbb{N}_{c-1},
\end{array}
$$

when $z=c$, we have from the assumption $\left({ }_{c-1}^{R} \nabla^{\varepsilon, \rho} \chi\right)(c)=\chi(c) \geq 0$.

Clearly, $\chi(c-1)=0$. So, we can start the induction from the next step.

Assume that $\left({ }_{c-1}^{R} \nabla^{\varepsilon, \rho} \chi\right)(i) \geq 0, \forall i<z$. We shall show that $\left({ }_{c-1}^{R} \nabla^{\varepsilon, \rho} \chi\right)(z) \geq 0$.

Since, from assumption, $\chi(z)$ is increasing, it follows that $\chi(z) \geq \chi(z-1) \geq \chi(c) \geq 0, \forall z \in \mathbb{N}_{c}$ :

$$
\begin{aligned}
\left({ }_{c-1}^{R} \nabla^{\varepsilon, \rho} \chi\right)(z)= & \chi(z)-\frac{\varepsilon}{\Gamma(1-\varepsilon)} \sum_{l=c}^{z-1} \rho^{z-\iota}(z-\varsigma(\iota))^{\overline{-\varepsilon-1}} \chi(\iota) \\
= & \chi(z)-\frac{\varepsilon}{\Gamma(1-\varepsilon)} \rho^{z-z+1}(z-\varsigma(z-1))^{\overline{-\varepsilon-1}} \chi(z-1) \\
& -\frac{\varepsilon}{\Gamma(1-\varepsilon)} \sum_{\iota=c}^{z-2} \rho^{z-\iota}(z-\varsigma(\iota))^{\overline{-\varepsilon-1}} \chi(\iota) \\
= & \chi(z)-\varepsilon \rho \chi(z-1)-\frac{\varepsilon}{\Gamma(1-\varepsilon)} \sum_{\iota=c}^{z-2} \rho^{z-\iota} \\
& \cdot(z-\varsigma(\iota))^{-\varepsilon-1} \chi(\iota) \\
& +\frac{\varepsilon}{\Gamma(1-\varepsilon)} \sum_{\iota=c}^{z-2} \rho^{z-\iota}(z-\varsigma(\iota))^{\overline{-\varepsilon-1}} \chi(z-1) \\
& -\frac{\varepsilon}{\Gamma(1-\varepsilon)} \sum_{l=c}^{z-2} \rho^{z-\iota}(z-\varsigma(\iota))^{\overline{-\varepsilon-1}} \chi(z-1) \\
= & \chi(z)-\varepsilon \rho \chi(z-1) \\
& +\frac{\varepsilon}{\Gamma(1-\varepsilon)} \sum_{\imath=c}^{z-2} \rho^{z-\iota}(z-\varsigma(\iota))^{\overline{-\varepsilon-1}}(\chi(z-1)-\chi(\iota))
\end{aligned}
$$

$$
\begin{aligned}
& -\frac{\varepsilon}{\Gamma(1-\varepsilon)} \sum_{\imath=c}^{z-2} \rho^{z-\iota}(z-\varsigma(\iota))^{\overline{-\varepsilon-1}} \chi(z-1) \\
\geq & \chi(z)-\varepsilon \rho \chi(z-1)-\frac{\varepsilon}{\Gamma(1-\varepsilon)} \sum_{\imath=c}^{z-2} \rho^{z-\iota} \\
& \cdot(z-\varsigma(\iota))^{\overline{-\varepsilon-1}} \chi(z-1) \\
= & \chi(z)-\frac{\varepsilon}{\Gamma(1-\varepsilon)} \sum_{\imath=c}^{z-1} \rho^{z-\iota} \\
& \cdot(z-\varsigma(\iota))^{\overline{-\varepsilon-1}} \chi(z-1) \\
= & \chi(z)-\chi(z-1)+\chi(z-1) \\
& -\frac{\varepsilon}{\Gamma(1-\varepsilon)} \sum_{\imath=c}^{z-2} \rho^{z-\iota}(z-\varsigma(\iota))^{\overline{-\varepsilon-1}} \chi(z-1)
\end{aligned}
$$$$
\geq \chi(z-1)-\frac{\varepsilon}{\Gamma(1-\varepsilon)} \sum_{l=c}^{z-1} \rho^{z-\iota}
$$$$
\cdot(z-\varsigma(\iota))^{\overline{-\varepsilon-1}} \chi(z-1)
$$$$
=\chi(z-1)\left(1-\frac{\varepsilon}{\Gamma(1-\varepsilon)} \sum_{l=c}^{z-1} \rho^{z-\iota}(z-\varsigma(\iota))^{\overline{-\varepsilon-1}}\right)
$$

$\geq 0$.

Theorem 3. Let $\chi: \mathbb{N}_{c-1} \longrightarrow \mathbb{R}$ be a function which satisfies $\chi(c)>0$ and be strictly increasing on $\mathbb{N}_{c}$, where $0<\varepsilon<1$ and $0<\rho \leq 1$. Then,

$$
\left({ }_{c-1}^{R} \nabla^{\varepsilon, \rho} \chi\right)>0, \quad z \in \mathbb{N}_{c-1} .
$$

Proof. Since

$$
\begin{aligned}
\left({ }_{c-1}^{R} \nabla^{\varepsilon, \rho} \chi\right)(z)= & \chi(z)-\frac{\varepsilon}{\Gamma(1-\varepsilon)} \sum_{l=c}^{z-1} \rho^{z-\iota} \\
& \cdot(z-\varsigma(\iota))^{\overline{-\varepsilon-1}} \chi(\iota), \quad z \in \mathbb{N}_{c-1},
\end{aligned}
$$

when $z=c$, we have $\left({ }_{c-1}^{R} \nabla^{\varepsilon, \rho} \chi\right)(c)=\chi(c)>0$. Clearly, $\chi(c-1)=0$, and so we can start the induction from the next step.

Assume that $\left({ }_{c-1}^{R} \nabla^{\varepsilon, \rho} \chi\right)(i)>0, \forall i<z$. We shall show that $\left(\begin{array}{l}R \\ c-1\end{array} \nabla^{\varepsilon, \rho} \chi\right)(z)>0$.

Since, from assumption, $\chi(z)$ is increasing it follows that $\chi(z)>\chi(z-1)>\chi(c)>0, \forall z \in \mathbb{N}_{c}$ : 


$$
\begin{aligned}
\left({ }_{c-1}^{R} \nabla^{\varepsilon, \rho} \chi\right)(z)= & \chi(z)-\frac{\varepsilon}{\Gamma(1-\varepsilon)} \sum_{l=c}^{z-1} \rho^{z-\iota}(z-\varsigma(\iota))^{\overline{-\varepsilon-1}} \chi(\iota) \\
> & \chi(z)-\chi(z-1)+\chi(z-1) \\
& -\frac{\varepsilon}{\Gamma(1-\varepsilon)} \sum_{\imath=c}^{z-1} \rho^{z-\iota}(z-\varsigma(\iota))^{\overline{-\varepsilon-1}} \chi(z-1) \\
> & \chi(z-1)-\frac{\varepsilon}{\Gamma(1-\varepsilon)} \sum_{l=c}^{z-1} \\
& \cdot \rho^{z-\iota}(z-\varsigma(\iota))^{\overline{-\varepsilon-1}} \chi(z-1) \\
= & \chi(z-1)\left(1-\frac{\varepsilon}{\Gamma(1-\varepsilon)} \sum_{l=c}^{z-1} \rho^{z-\iota}(z-\varsigma(\iota))^{-\varepsilon-1}\right)>0
\end{aligned}
$$

Theorem 4. Let $\chi: \mathbb{N}_{c-1} \longrightarrow \mathbb{R}$ be a function, and suppose that $\left({ }_{c-1}^{R} \nabla^{\varepsilon, \rho} \chi\right)(z) \leq 0$ for $0<\varepsilon<1$ and $0<\rho \leq 1, z \in \mathbb{N}_{c-1}$. Then, $\chi(z)$ is $\varepsilon \rho-$ decreasing.

Proof. Let $\theta: \mathbb{N}_{c-1} \longrightarrow \mathbb{R}$ be a function such that $\theta(z)=-\chi(z)$; hence,

$$
\left({ }_{c-1}^{R} \nabla^{\mathcal{\varepsilon}, \rho} \theta\right)(z)=\left({ }_{c-1}^{R} \nabla^{\mathcal{E}, \rho}(-\chi)\right)(z)=-\left({ }_{c-1}^{R} \nabla^{\mathcal{E}, \rho} \chi\right)(z) \geq 0 .
$$

Now by Theorem 1 , we conclude that $\theta(z)$ is $\varepsilon \rho-$ increasing.

Hence,

$$
\theta(z+1) \geq \varepsilon \rho \theta(z)
$$

which is

$$
\begin{gathered}
-\chi(z+1) \geq \varepsilon \rho(-\chi(z)), \\
\chi(z+1) \leq \varepsilon \rho \chi(z),
\end{gathered}
$$

that is to say, $\chi(z)$ is $\varepsilon \rho-$ decreasing.

Theorem 5. Let a function $\chi: \mathbb{N}_{c-1} \longrightarrow \mathbb{R}$ be decreasing on $\mathbb{N}_{c}$ such that $\chi(c) \leq 0$. Then, for $0<\varepsilon<1$ and $0<\rho \leq 1$, we have

$$
\left({ }_{c-1}^{R} \nabla^{\varepsilon, \rho} \chi\right)(z) \leq 0, \quad \forall z \in \mathbb{N}_{c-1}
$$

Proof. The proof follows by applying Theorem 2 to $\theta(z)=-\chi(z)$.

Using Theorem 4.3 in [4] we can state the following.

Theorem 6 (see [4]). For any $0<\varepsilon<1, \quad 0<\rho \leq 1$, $p=(\rho-1 / \rho)$, and $\chi: \mathbb{N}_{c+1} \longrightarrow \mathbb{R}$, the following equality holds:

$$
\left({ }_{c}^{R} \nabla^{-\varepsilon, \rho}{ }_{c-1}^{R} \nabla^{\varepsilon, \rho} \chi\right)(z)=\chi(z)-\frac{\widehat{e}_{p}(z, c)}{\Gamma(\varepsilon)}(z-c+1)^{\overline{\varepsilon-1}} \chi(c) .
$$

\section{Application: Mean Value Theorem (MVT)}

First, for the sake of simplification, depending on Theorem 6, we shall write

$$
\left({ }_{c}^{R} \nabla^{-\varepsilon, \rho}{ }_{c-1}^{R} \nabla^{\mathcal{E}, \rho} \chi\right)(z)=\chi(z)-S(z, c) \chi(c),
$$

where

$$
S(z, c)=\frac{\widehat{e}_{p}(z, c)}{\Gamma(\varepsilon)}(z-c+1)^{\overline{\varepsilon-1}}
$$

Theorem 7 (the fractional proportional difference MVT)

Let $\Theta$ and $\theta$ be functions defined on $\mathbb{N}_{c} \cap{ }_{d} \mathbb{N}=\{c, c+1, c+2, \ldots, d-2, d-1, d\}$, where $c \equiv d \bmod 1$. Assume that $\theta$ is strictly increasing, $\theta(c)>0$, and $0<\varepsilon<1$ and $0<\rho \leq 1$. Then, there exist $s_{1}, s_{2} \in \mathbb{N}_{c} \cap{ }_{d} \mathbb{N}$ such that

$$
\frac{\left(\begin{array}{l}
R \\
c-1
\end{array} \nabla^{\varepsilon, \rho} \Theta\right)\left(s_{1}\right)}{\left({ }_{c-1}^{R} \nabla^{\mathcal{\varepsilon}, \rho} \theta\right)\left(s_{1}\right)} \leq \frac{\Theta(d)-S(d, c) \Theta(c)}{\theta(d)-S(d, c) \theta(c)} \leq \frac{\left({ }_{c-1}^{R} \nabla^{\mathcal{\varepsilon}, \rho} \Theta\right)\left(s_{2}\right)}{\left({ }_{c-1}^{R} \nabla^{\mathcal{E}, \rho} \theta\right)\left(s_{2}\right)} .
$$

Proof. First we need to show that $\theta(d)-S(d, c) \theta(c)>0$. Since $\theta$ is strictly increasing, then by Theorem 3 we have

$$
\left({ }_{c-1}^{R} \nabla^{\mathcal{\varepsilon}, \rho} \theta\right)(z)>0, \quad \forall z, \in \mathbb{N}_{c} \cap{ }_{d} \mathbb{N} .
$$

Applying the fractional sum operator associated to $\left({ }_{c}^{R} \nabla^{\varepsilon, \rho} \theta\right)(z)$ on both sides of the inequality, by means of $(40)$, we get

$$
{ }_{c}^{R} \nabla^{-\mathcal{\varepsilon}, \rho}\left({ }_{c-1}^{R} \nabla^{\mathcal{\varepsilon}, \rho} \theta\right)(z)>\left({ }_{c-1}^{R} \nabla^{-\varepsilon, \rho}(0)\right) \quad \forall z, \in \mathbb{N}_{c} \cap{ }_{d} \mathbb{N},
$$

or we have

$$
\theta(z)-S(z, c) \theta(c)>0 . \quad \forall z \in \mathbb{N}_{c} \cap{ }_{d} \mathbb{N} .
$$

For $z=d$, we get

$$
\theta(d)-S(d, c) \theta(c)>0 .
$$

To prove the theorem, we use the proof by contradiction. Assume (42) is not true, then either

$$
\frac{\Theta(d)-S(d, c) \Theta(c)}{\theta(d)-S(d, c) \theta(c)}<\frac{\left(\begin{array}{l}
R-1 \\
c-1
\end{array} \nabla^{\mathcal{\varepsilon}, \rho} \Theta\right)(z)}{\left({ }_{c-1}^{R} \nabla^{\varepsilon, \rho} \theta\right)(z)}, \quad \forall z \in \mathbb{N}_{c} \cap{ }_{d} \mathbb{N}
$$

or

$$
\frac{\Theta(d)-S(d, c) \Theta(c)}{\theta(d)-S(d, c) \theta(c)}>\frac{\left(\begin{array}{l}
R-1 \\
c-1
\end{array} \nabla^{\mathcal{\varepsilon}, \rho} \Theta\right)(z)}{\left({ }_{c-1}^{R} \nabla^{\varepsilon, \rho} \theta\right)(z)}, \quad \forall z \in \mathbb{N}_{c} \cap{ }_{d} \mathbb{N}
$$

Again, since $\theta$ is strictly increasing, then by Theorem 3 we conclude that

$$
\left({ }_{c-1}^{R} \nabla^{\varepsilon, \rho} \Theta\right)(z), \quad \forall z \in \mathbb{N}_{c} \cap{ }_{d} \mathbb{N} .
$$

Hence, (47) becomes 


$$
\begin{array}{r}
\frac{\Theta(d)-S(d, c) \Theta(c)}{\theta(d)-S(d, c) \theta(c)}\left({ }_{c-1}^{R} \nabla^{\varepsilon, \rho} \theta\right)(z)<\left({ }_{c-1}^{R} \nabla^{\varepsilon, \rho} \Theta\right)(z), \\
\forall z \in \mathbb{N}_{c} \cap{ }_{d} \mathbb{N} .
\end{array}
$$

Applying the fractional sum operator on both sides of the inequality at $z=d$ and by making use of (43), we see that

$$
\begin{aligned}
& \frac{\Theta(d)-S(d, c) \Theta(c)}{\theta(d)-S(d, c) \theta(c)}(\theta(d)-S(d, c) \theta(c)) \\
& \quad<(\Theta(d)-S(d, c) \Theta(c)),
\end{aligned}
$$

and hence, $\Theta(d)<\Theta(d)$, which is a contradiction. In a similar way, (48) leads to contradiction.

\section{Conclusions}

The conclusions of this article are summarized as follows:

(1) The summation and difference of a discrete fractional proportional have been detected.

(2) The nabla discrete new Riemann-Liouville and Caputo fractional proportional differences of order $0<\varepsilon<1$ on the time scale $\mathbb{Z}$ have been formulated.

(3) The fractional proportional sums associated to $\left({ }_{c}^{R} \nabla^{\varepsilon, \rho} \chi\right)(z)$ with order $0<\varepsilon<1$ have been defined.

(4) The relation between nabla Riemann-Liouville and Caputo fractional proportional differences has been detected.

(5) The monotonicity results for the nabla Caputo fractional proportional difference which are if $\left({ }_{c-1}^{R} \nabla^{\varepsilon, \rho} \chi\right)(z)>0$, then $\chi(z)$ is $\varepsilon \rho$-increasing; if $\chi(z)$ is strictly increasing on $\mathbb{N}_{c}$ and $\chi(c)>0$, then $\left({ }_{c-1}^{R} \nabla^{\varepsilon, \rho} \chi\right)(z)>0$ has been proved as well.

(6) A new version of the fractional proportional difference of the mean value theorem on $\mathbb{Z}$ has been proved as an application.

\section{Data Availability}

No data were used to support this study.

\section{Conflicts of Interest}

The authors declare that they have no conflicts of interest.

\section{Authors' Contributions}

All the authors participated in obtaining the main results of this manuscript and drafted the manuscript. All authors read and approved the final manuscript.

\section{Acknowledgments}

The corresponding author would like to thank Prince Sultan University for funding this work through the research group Nonlinear Analysis Methods in Applied Mathematics (NAMAM), group number RG-DES-2017-01-17.

\section{References}

[1] I. Podlubny, Fractional Differential Equations, Academic Press, San Diego, CA, USA, 1999.

[2] T. Abdeljawad, "Different type kernel h-fractional differences and their fractional h-sums," Chaos, Solitons \& Fractals, vol. 2018, no. 116, pp. 146-156, 2018.

[3] I. Suwan, T. Abdeljawad, and F. Jarad, "Monotonicity analysis for nabla h-discrete fractional Atangana-Baleanu differences," Chaos, Solitons \& Fractals, vol. 117, pp. 50-59, 2018.

[4] T. Abdeljawad, F. Jarad, and J. Alzabut, "Fractional proportional differences with memory," The European Physical Journal Special Topics, vol. 226, no. 16-18, pp. 3333-3354, 2017.

[5] F. Bozkurt, T. Abdeljawad, and M. Hajji, "Stability analysis of a fractional order differential equation model of a brain tumor growth depending on the density," Applied Mathematics and Computation, vol. 14, no. 1, pp. 50-62, 2015.

[6] H. Sun, Y. Zhang, D. Baleanu, W. Chen, and Y. Chen, "A new collection of real world applications of fractional calculus in science and engineering," Communications in Nonlinear Science and Numerical Simulation, vol. 64, pp. 213-231, 2018.

[7] A. Atangana and J. Aguilar, "Decolonisation of fractional calculus rules: breaking commutativity and associativity to capture more natural phenomena," The European Physical Journal Plus, vol. 133, no. 4, pp. 1-22, 2018.

[8] D. Kumar, J. Singh, D. Baleanu, and Sushila, "Analysis of regularized long-wave equation associated with a new fractional operator with Mittag-Leffler type kernel," Physica A: Statistical Mechanics and Its Applications, vol. 492, pp. 155167, 2018.

[9] J. Prez, J. Gmez-Aguilar, D. Baleanu, and F. Tchier, "Chaotic attractors with fractional conformable derivatives in the LiouvilleCaputo sense and its dynamical behaviors," Entropy, vol. 20, no. 5, 2018.

[10] A. Babiarz, A. Lgowski, and M. Niezabitowski, Robot Path Control with Al-Alaoui Rule for Fractional Calculus Discretization, Theory and Applications of Non-integer Order Systems, pp. 405-418, Springer, Cham, Switzerland, 2017.

[11] A. Babiarz, T. Grzejszczak, A. Lgowski, and M. Niezabitowski, "On inverse kinematics with fractional calculus," in Proceedings of the Asia-Pacific Conference on Intelligent Robot Systems (ACIRS) 2016, pp. 127-131, IEEE, Tokyo, Japan, July 2016.

[12] K. Saad, A. Atangana, and D. Baleanu, "New fractional derivatives with non-singular kernel applied to the Burgers equation," Chaos, vol. 28, 2018.

[13] D. Baleanu, J. Asad, and A. Jajarmi, "The fractional model of spring pendulum: new features within different kernels," Proceedings of the Romanian Academy, Series A, vol. 19, no. 3, pp. 447-454, 2018.

[14] A. Kilbas, M. Srivastava, and J. Trujillo, "Theory and application of fractional differential equations," North Holland Mathematics Studies, vol. 204, 2006.

[15] F. M. Atici and P. Eloe, "Discrete fractional calculus with the nabla operator," Electronic Journal of Qualitative Theory of Differential Equations, vol. 2009, no. 3, pp. 1-12, 2009.

[16] C. Goodrich and A. C. Peterson, Discrete Fractional Calculus, Springer, Cham, Switzerland, 2015.

[17] F. Jarad, T. Abdeljawad, and D. Baleanu, "Fractional variational optimal control problems with delayed arguments," Nonlinear Dynamics, vol. 62, no. 3, pp. 609-614, 2010. 
[18] F. Atici and M. Uyanik, "Analysis of discrete fractional operators," Applicable Analysis and Discrete Mathematics, vol. 9, no. 1, pp. 139-149, 2015.

[19] L. Erbe, C. Goodrich, B. Jia, and A. Peterson, "Monotonocity results for delta and nabla fractional differences revisited," Mathematica Slovaca, vol. 67, no. 4, pp. 895-906, 2017.

[20] C. S. Goodrich, "A convexity result for fractional differences," Applied Mathematics Letters, vol. 35, pp. 58-62, 2014.

[21] R. Dahal and C. S. Goodrich, "An almost sharp monotonicity result for discrete sequential fractional delta differences," Journal of Difference Equations and Applications, vol. 23, no. 7, pp. 1190-1203, 2017.

[22] T. Abdeljawad and D. Baleanu, "Monotonicity analysis of a nabla discrete fractional operator with discrete Mittag-Leffler kernel," Chaos, Solitons \& Fractals, vol. 102, pp. 106-110, 2017.

[23] T. Abdeljawad, "Dual identities in fractional difference calculus within Riemann," Advances in Difference Equations, vol. 2013, no. 1, 2013.

[24] T. Abdeljawad, "On delta and nabla Caputo fractional differences and dual Identities," Discrete Dynamics in Nature and Society, vol. 2013, Article ID 406910, 12 pages, 2013.

[25] T. Abdeljawad and B. Abdalla, "Monotonicity results for delta and nabla Caputo and Riemann fractional differences via dual identities," Filomat, vol. 31, no. 12, pp. 3671-3683, 2017.

[26] A. Atangana and D. Baleanu, "New fractional derivatives with nonlocal and non-singular kernel: theory and application to heat transfer model," Thermal Science, vol. 20, no. 2, pp. 763-769, 2016.

[27] P. Ostalczyk, Discrete Fractional Calculus, Applications in Control and Image Processing, Series in Computer Vision, vol. 4, World Scientific Publishing, Singapore, 2016.

[28] M. Holm, The Theory of Discrete Fractional Calculus: Development and Application, University of Nebraska-Lincoln, Lincoln, Nebraska, 2011.

[29] M. Ganji and F. Gharari, "An application of discrete fractional calculus in statistics," Revista Investigation Operacional, vol. 38, no. 3, pp. 272-280, 2017.

[30] T. Abdeljawad and D. Baleanu, "On fractional derivatives with exponential kernel and their discrete versions," Reports on Mathematical Physics, vol. 80, no. 1, pp. 11-27, 2017.

[31] T. Abdeljawad and D. Baleanu, "Discrete fractional differences with non-singular discrete Mittag-Leffler kernels," Advances in Difference Equations, vol. 2016, p. 232, 2016.

[32] T. Abdeljawad and D. Baleanu, "Monotonicity results for fractional difference operators with discrete exponential kernels," Advances in Difference Equations, vol. 2017, no. 78, 2017.

[33] I. Suwan, S. Owies, and T. Abdeljawad, "Monotonicity results for $h$-discrete fractional operators and application," Advances in Difference Equations, vol. 2018, p. 207, 2018. 